J. Dairy Sci. 98:1563-1570

http://dx.doi.org/10.3168/jds.2014-8828

(C) American Dairy Science Association ${ }^{\circledR}, 2015$.

\title{
Rapid detection of Staphylococcus aureus in dairy and meat foods by combination of capture with silica-coated magnetic nanoparticles and thermophilic helicase-dependent isothermal amplification
}

\author{
Xingxing Chen, ${ }^{* 1}$ Xiaoli Wu, $\dagger^{1}$ Min Gan, ${ }^{*}$ Feng Xu, ${ }^{*}$ Lihua He, ${ }^{*}$ Dong Yang, ${ }^{*}$ Hengyi Xu, ${ }^{*}$ Nagendra P. Shah, $\ddagger^{2}$ \\ and Hua Wei*2 \\ *State Key Laboratory of Food Science and Technology, Nanchang University, Nanchang, 330047, China \\ †School of Basic Medical Science, Jiangxi University of Chinese Traditional Medicine, Nanchang, Jiangxi, 330000, China \\ ¥Food and Nutritional Science, School of Biological Sciences, The University of Hong Kong, Pokfulam Road, Hong Kong
}

\begin{abstract}
Staphylococcus aureus is one of the main pathogens in dairy and meat products; therefore, developing a highly sensitive and rapid method for its detection is necessary. In this study, a quantitative detection method for Staph. aureus was developed using silica-coated magnetic nanoparticles and thermophilic helicase-dependent isothermal amplification. First, genomic DNA was extracted from lysed bacteria using silica-coated magnetic nanoparticles and amplified using thermophilic helicase-dependent isothermal amplification. After adding the nucleic-acid dye SYBR Green I to the amplicons, the fluorescence intensity was observed using a UV lamp or recorded using a fluorescence spectrophotometer. This detection system had a detection limit of $5 \times 10^{0} \mathrm{cfu} / \mathrm{mL}$ in pure culture and milkpowder samples and $5 \times 10^{1} \mathrm{cfu} / \mathrm{mL}$ in pork samples using a UV light in less than $2 \mathrm{~h}$. In addition, a good linear relationship was obtained between fluorescence intensity and bacterial concentrations ranging from $10^{2}$ to $10^{4} \mathrm{cfu} / \mathrm{mL}$ under optimal conditions. Furthermore, the results from contaminated milk powder and pork samples suggested that the detection system could be used for the quantitative analysis of Staph. aureus and applied potentially to the food industry for the detection of this pathogen.
\end{abstract}

Key words: Staphylococcus aureus, silica-coated magnetic nanoparticles, thermophilic helicase-dependent isothermal amplification, SYBR Green I

\section{INTRODUCTION}

Food-borne diseases caused by pathogens have attracted worldwide attention because almost a quarter

Received September 6, 2014.

Accepted November 11, 2014.

${ }^{1}$ These authors contributed equally.

${ }^{2}$ Corresponding authors: weihua114@live.cn and npshah@hku.hk of the population is currently at risk of suffering from food-borne illnesses (Omiccioli et al., 2009). Staphylococcus aureus is the second-most-prevalent food-borne pathogen that exists widely in the environment, particularly in food (Miao et al., 2010). A previous study reported that Staph. aureus accounted for $2.57 \%$ of the $9,388,075$ food-borne illnesses per year in the United States (Scallan et al., 2011). In addition, reports suggest that about 30 to $50 \%$ of the population carries Staph. aureus (Lowy, 1998). Because of its prevalence and the associated hazard, a rapid and accurate method to detect Staph. aureus is crucial to prevent severe health problems.

The conventional standardized methods used to detect and identify Staph. aureus were based on cell culture and biochemical tests, including enrichment cultivation, selective cultivation, biochemical identification, and serological reactions. However, the practical applications of conventional methods are limited because they are laborious and time consuming (Gilbert, 2002). Recently, molecular diagnostics involving nucleic-acid amplification have become the most commonly used methods for bacterial detection (Lazcka et al., 2007). Polymerase chain reaction, a nucleic acid-based method, is used widely to detect Staph. aureus (Kim et al., 2001; Vancraeynest et al., 2007; Yang et al., 2007) because of its high sensitivity and specificity. Nevertheless, PCR normally requires precise temperature control during thermal cycling and a time-consuming temperature-ramping process (Wang et al., 2011). Furthermore, extensive sample preparation, specialized instruments, and technical expertise are needed.

Consequently, isothermal amplification techniques have attracted increasing attention in recent years and have been used successfully as an alternative tool for the rapid detection of pathogens (Gill and Ghaemi, 2008; Kim and Easley, 2011). Examples of such techniques include strand displacement amplification (Hellyer and Nadeau, 2004), rolling circle amplification (Haible et al., 2006), loop-mediated isothermal amplification 
(Li et al., 2013; Yang et al., 2013), and nucleic acid sequence-based amplification (Mollasalehi and Yazdanparast, 2013). Compared with PCR-based methods, isothermal nucleic-acid amplification has the potential to achieve low-cost molecular diagnosis because it is performed at a constant temperature, thereby eliminating the requirement for a thermocycler. However, most isothermal nucleic acid-amplification techniques still have limitations such as complicated reaction schemes and challenges amplifying DNA targets of sufficient length to be useful for various applications and diagnosis (Wang et al., 2011). Therefore, thermophilic helicase-dependent isothermal amplification (tHDA), a relatively novel isothermal amplification technology developed by BioHelix (Beverly, MA; Vincent et al., 2004) has been introduced. In the past $10 \mathrm{yr}$, tHDA has been used successfully to detect several pathogens, including Escherichia coli (Mahalanabis et al., 2010), Helicobacter pylori (Gill et al., 2008), Chlamydia trachomatis, and Neisseria gonorrhoeae (Doseeva et al., 2011). However, tHDA-based methods have not yet been used to monitor target pathogens quantitatively.

In this study, we used tHDA to detect Staph. aureus quantitatively using nucleic-acid dye SYBR Green I (Invitrogen, Shanghai, China) as the signal reporter based on its high specificity for double-stranded DNA (dsDNA) and bright fluorescence upon intercalation into dsDNA. In addition, silica-coated magnetic nanoparticles (Si-MNP) were used to extract genomic DNA from food samples, which successfully avoided sample preincubation, and eliminated the influence of the food matrix. This platform could detect Staph. aureus even at low levels and exhibited specificity for this pathogen.

\section{MATERIALS AND METHODS}

\section{Materials and Reagents}

Silica-coated magnetic nanoparticles were provided by Wuxi Zodoboer Biotech. Co. Ltd. (Wuxi, China). The nucleic-acid dye SYBR Green I was obtained from Invitrogen. The DNA marker was purchased from Dalian TaKaRa Biotechnology Company Ltd. (Dalian, China). All culture media were purchased from Beijing LuQiao Company (Beijing, China). All other reagents were of analytical grade and were purchased from Sigma Chemical Co. Ltd. (St. Louis, MO).

\section{Strains and Cultivation}

The strains used in this study are listed in Table 1. All strains were provided by the State Key Laboratory of Food Science and Technology, Nanchang Univer- sity (Nanchang, China), and stored in $20 \%$ glycerol at $-80^{\circ} \mathrm{C}$. The Staph. aureus strains were cultured in $\mathrm{Lu}-$ ria-Bertani broth at $37^{\circ} \mathrm{C}$, and the remaining bacteria were cultured according to the growth requirements of the individual organisms. Bacterial cells were harvested and washed twice with phosphate buffer (PBS, 0.01 $M, \mathrm{pH}$ 7.4). The concentration of Staph. aureus was determined using colony counting.

\section{Preparation of Staph. aureus-Spiked Samples}

The whole milk powder and pork samples, which were free of Staph. aureus, were purchased from a local supermarket. One-gram aliquots of Staph. aureus-negative whole milk powder were reconstituted in $8 \mathrm{~mL}$ of sterile distilled water, and then 1-mL aliquots of serial 10-fold dilutions of Staph. aureus were added to the reconstituted whole milk powder to obtain concentrations ranging from $5 \times 10^{0}$ to $5 \times 10^{7} \mathrm{cfu} / \mathrm{mL}$. Moreover, to evaluate the sensitivity of the method in the presence of non-Staph. aureus bacteria, $10^{8} \mathrm{cfu} / \mathrm{mL}$ of each of Escherichia coli, Listeria monocytogenes, and Lactobacillus plantarum was also mixed into the reconstituted whole milk powder containing $5 \times 10^{0}$ to $5 \times 10^{7} \mathrm{cfu} /$ $\mathrm{mL}$ Staph. aureus.

For pork samples, serially diluted Staph. aureus and $10^{8} \mathrm{cfu} / \mathrm{mL}$ E. coli, L. monocytogenes, and L. plantarum were mixed with a minced sample of meat to a final meat concentration of $10 \%$ (wt/vol). Then minced pork samples containing different concentrations of Staph. aureus (ranging from $5 \times 10^{0}$ to $5 \times 10^{7} \mathrm{cfu} / \mathrm{mL}$ ) were also obtained and used for DNA extraction. The concentration of Staph. aureus was determined using colony counting.

\section{Genomic DNA Extraction Using Si-MNP}

All DNA extractions were performed as previously described previously (Chen et al., 2014a) with some modifications. Briefly, 1-mL aliquots of Staph. aureus sample were boiled at $100^{\circ} \mathrm{C}$ for $10 \mathrm{~min}$ to release the genomic DNA completely. Subsequently, $10 \mu \mathrm{L}$ of SiMNP solution $(10 \mathrm{mg} / \mathrm{mL})$ and $500 \mu \mathrm{L}$ of hydrosaline solution $(3 \mathrm{M} \mathrm{NaCl}, 2 \mathrm{M} \mathrm{KCl})$ were added into the tubes. The tubes were then incubated for $20 \mathrm{~min}$ at $24^{\circ} \mathrm{C}$ with gentle shaking (130 rpm), and the Si-MNPDNA complexes were isolated magnetically from the medium by placing the tubes in the separator racks on a permanent magnet $(0.4 \mathrm{~T})$ for 3 min. After washing twice with $80 \%$ ethanol, $20 \mu \mathrm{L}$ of sterile deionized water was added to dissolve the DNA adsorbed on the surface of Si-MNP. Finally, the Si-MNP in the suspensions were collected again using the magnetic separator, and the supernatants containing the desorbed DNA were 
obtained and stored at $-20^{\circ} \mathrm{C}$ before amplification. All experiments were repeated in triplicate.

\section{Staph. aureus Primers and the Capture Efficiency of Si-MNP}

The primers used to amplify Staph. aureus were as follows: sau-F, 5'-CAAGATGTGCACAGTTACTTACACATATG-3', and sau-R, 5'-CACTTATAGATGGATCCGCGCT-3'. They were designed based on the conserved $16 \mathrm{~S}$ rRNA gene sequences identified in GenBank, and their specificity for Staph. aureus was verified previously (Chen et al., 2014b).

To evaluate the capture efficiency of the Si-MNP, real-time PCR was performed on a $7900 \mathrm{HT}$ fast realtime PCR system (Applied Biosystems, Foster City, CA) using a SYBR Green PCR kit (TaKaRa) and performed in a $20-\mu \mathrm{L}$ reaction mixture containing $10 \mu \mathrm{L}$ of $2 \times$ SYBR Premix Ex Taq II buffer, $1 \mu \mathrm{L}(0.1 \mathrm{n} M)$ of primer sau-F/R primers, and $2.5 \mu \mathrm{L}$ of DNA templates, and the volume was adjusted by using sterile deionized water. Cycling parameters were as follows: an initial denaturation at $95^{\circ} \mathrm{C}$ for $10 \mathrm{~min}, 42$ cycles of $95^{\circ} \mathrm{C}(5$ s), $62^{\circ} \mathrm{C}(60 \mathrm{~s})$, and $72^{\circ} \mathrm{C}(20 \mathrm{~s})$, and a final elongation at $72^{\circ} \mathrm{C}$ for $10 \mathrm{~min}$. Each sample was run in triplicate, including a blank with no target DNA. The assays were run in triplicate.

\section{tHDA}

The tHDA reactions were performed using a commercially available kit (IsoAmp II Universal tHDA Kit, BioHelix). The one-step 50- $\mu \mathrm{L}$ tHDA reaction contained $5 \mu \mathrm{L}$ of $10 \times$ annealing buffer; $2 \mu \mathrm{L}$ of $\mathrm{MgSO}_{4}(100$ $\mathrm{m} M), 4 \mu \mathrm{L}$ of $\mathrm{NaCl}(500 \mathrm{mM}), 3.5 \mu \mathrm{L}$ of IsoAmp:emoji: dNPT solution, $0.75 \mu \mathrm{L}$ of each forward and reverse primer $(5 \mu M), 3.5 \mu \mathrm{L}$ of IsoAmp:emoji: enzyme mix, and $1 \mu \mathrm{L}$ of test template. The volume was adjusted using sterile deionized water. All reaction mixtures were covered with $50 \mu \mathrm{L}$ of mineral oil to avoid evaporation (Picard et al., 2005), and the tubes were placed on ice for $1 \mathrm{~min}$. The reaction was incubated at $65^{\circ} \mathrm{C}$ for 90 $\min$.

\section{Assay Procedure}

The assay used for the fluorescent detection of the target pathogen is illustrated in Figure 1. One milliliter of contaminated Staph. aureus samples was treated with $10 \mu \mathrm{L}$ of Si-MNP solution $(10 \mathrm{mg} / \mathrm{mL})$ to purify and enrich the target genomic DNA, and $20 \mu \mathrm{L}$ of the target Staph. aureus gene was then amplified using tHDA according to the manufacturer's protocol. Subsequently, $6 \mu \mathrm{L}$ of SYBR Green I (19.6 $\mathrm{mM}$ ) was added to the
Table 1. The bacterial strains used in experiment and results of specificity of the protocol

\begin{tabular}{|c|c|c|}
\hline No. & Species and strain & $\begin{array}{l}\text { Detected } \\
\text { by tHDA }\end{array}$ \\
\hline 1 & Staphylococcus aureus PSAV0001 & + \\
\hline 2 & Staph. aureus PSAV0002 & + \\
\hline 3 & Staph. aureus PSAV0007 & + \\
\hline 4 & Staph. aureus PSAV0012 & + \\
\hline 5 & Staph. aureus PSAV0021 & + \\
\hline 6 & Staph. aureus PSAV0024 & + \\
\hline 7 & Staph. aureus PSAV0027 & + \\
\hline 8 & Staph. aureus PSAV0035 & + \\
\hline 9 & Staph. aureus PSAV0040 & + \\
\hline 10 & Staph. aureus PSAV0202 & + \\
\hline 11 & Staph. aureus PSAV0205 & + \\
\hline 12 & Staph. aureus PSAV0207 & + \\
\hline 13 & Staph. aureus PSAV0209 & + \\
\hline 14 & Staph. aureus PSAV0210 & + \\
\hline 15 & Staph. aureus PSAV0213 & + \\
\hline 16 & Staph. aureus PSAV0214 & + \\
\hline 17 & Staph. aureus PSAV0216 & + \\
\hline 18 & Staph. aureus PSAV0218 & + \\
\hline 19 & Staph. aureus PSAV0219 & + \\
\hline 20 & Staph. aureus PSAV0220 & + \\
\hline 21 & Staph. aureus CMCC26001 & + \\
\hline 22 & Staph. aureus CMCC26002 & + \\
\hline 23 & Staph. aureus CMCC26003 & + \\
\hline 24 & Salmonella Typhimurium ATCC 13311 & - \\
\hline 25 & Pseudomonas aeruginosa CMCC10104 & - \\
\hline 26 & Salmonella Enteritidis CMCC 50041 & - \\
\hline 27 & Shigella flexneri ATCC29903 & - \\
\hline 28 & Escherichia coli O157:H7 PELI1264 & - \\
\hline 29 & E. coli O157:H7 PELI0480 & - \\
\hline 30 & Cronobacter sakazakii ATCC 29544 & - \\
\hline 31 & Cronobacter spp. CICC 21544 & - \\
\hline 32 & Shigella sonnei ATCC 29930 & - \\
\hline 33 & Listeria monocytogenes CMCC54001 & - \\
\hline 34 & L. monocytogenes PLMO1448 & - \\
\hline 35 & L. monocytogenes PLMO1344 & - \\
\hline 36 & Lactobacillus plantarum ATCC 8014 & - \\
\hline 37 & Lactobacillus acidophilus ATCC 4356 & - \\
\hline 38 & Enterococcus faecalis ATCC 19433 & - \\
\hline 39 & Campylobacter jejuni NC002163 & - \\
\hline 40 & Vibrio parahaemolyticus 1616 & - \\
\hline 41 & Bifidobacterium breve WBBR04 & - \\
\hline 42 & Bifidobacterium animalis WBBR05 & - \\
\hline 43 & Bifidobacterium bifidum WBBI01 & - \\
\hline 44 & Bifidobacterium longum WBLO01 & - \\
\hline 45 & Bifidobacterium infantis WBAN07 & - \\
\hline 46 & Bifidobacterium adolescentis WBAD08 & - \\
\hline 47 & Bifidobacterium lactis WLABO9 & - \\
\hline 48 & Lactobacillus rhamnosus GG ATCC 7469 & - \\
\hline 49 & Lactobacillus bulgaricus F1 & - \\
\hline 50 & Candida albicans $\mathrm{Z} 1$ & - \\
\hline
\end{tabular}

${ }^{1}$ tHDA $=$ thermophilic helicase-dependent isothermal amplification; + indicates positive signal; - indicates negative signal.

amplification product, incubated for target nucleic-acid detection, and then detected qualitatively under a UV light.

For quantitative detection, specific parameters, such as the concentration of SYBR Green I $(5,6,7,8,9$, and $10 \mu \mathrm{L})$, incubation time $(1,3,5,7$, and $9 \mathrm{~min})$, and amplification time $(50,60,70,80$, and $90 \mathrm{~min})$ were optimized. The fluorescence intensities of 3 replicates 


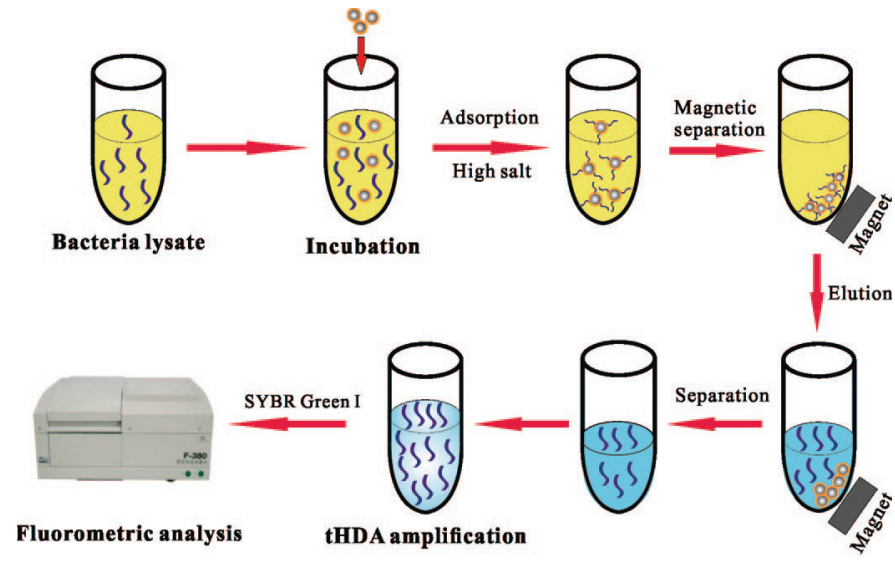

Si-MNP $\quad S$ double-stranded DNA

Figure 1. Overall process of Si-MNP-tHDA-SYBR Green I assay for detection of Staphylococcus aureus. Si-MNP = silica-coated magnetic nanoparticles; tHDA = thermophilic helicase-dependent isothermal amplification. SYBR Green I (Invitrogen, Shanghai, China). Color version available online.

for each target bacterial concentration were collected using an F-380 spectrophotometer (Shimadzu, Tokyo, Japan) equipped with a 150-W xenon lamp (Ushio Inc., Tokyo, Japan) with excitation at $495 \mathrm{~nm}$ and emission at $520 \mathrm{~nm}$. A calibration curve was constructed by plotting the fluorescence value against the Staph. aureus concentrations obtained under the optimized conditions. The amount of Staph. aureus in the milk powder and pork samples was calculated using the formula $R$ $=C / A$, where $R$ is the recovery of Staph. aureus, $C$ is the concentration of Staph. aureus determined using the calibration curve, and $A$ is the adsorption efficiency of the Si-MNP. The experiments were carried out in triplicate.

\section{Statistical Analysis}

Results were analyzed using SPSS 13.0 software (SPSS Inc., Chicago, IL) by means of independent oneway ANOVA tests in each sampling point. The differences among the 3 groups were assessed by means of the least significant difference multiple comparison test $(P<0.05)$.

\section{RESULTS AND DISCUSSION}

\section{Capture Efficiency of the Si-MNP for Staph. aureus Genomic DNA}

To evaluate the ability of Si-MNP to capture target genomic DNA in spiked samples, different concentrations of spiked samples containing Staph. aureus (rang-

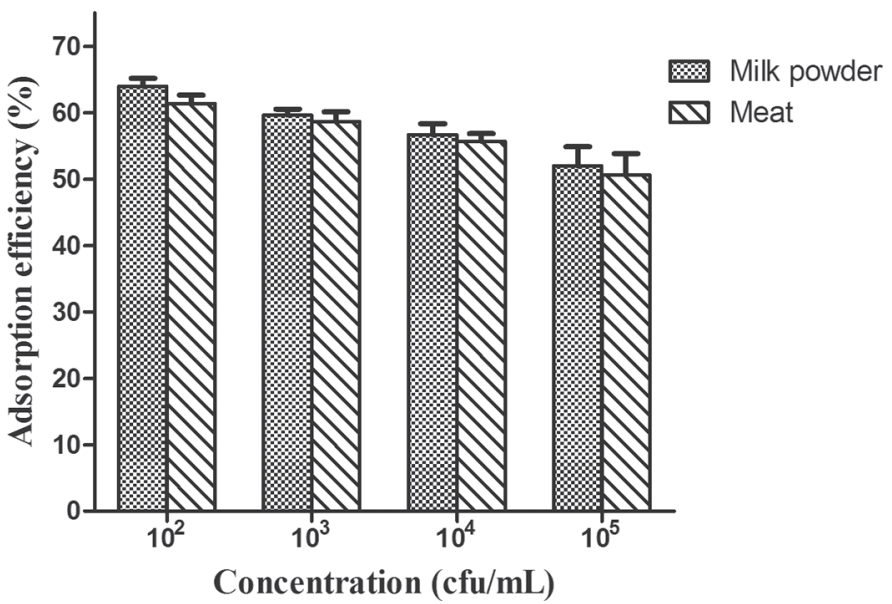

Figure 2. Real-time PCR evaluation of the adsorption efficiency of the silica-coated magnetic nanoparticles in milk powder and pork samples (cell counts of Staphylococcus aureus ranging from $5 \times 10^{2}$ to $\left.5 \times 10^{5} \mathrm{cfu} / \mathrm{mL}\right)$. Error bars indicated the deviation range in triplicate test results $(P>0.05)$.

ing from $10^{2}$ to $10^{5} \mathrm{cfu} / \mathrm{mL}$ ) were used to capture Staph. aureus genomic DNA, which was then analyzed using real-time PCR. As shown in Figure 2, more than 50\% of the Staph. aureus DNA fragments were recovered by Si-MNP in the spiked milk powder and pork samples. The recovery rate of DNA decreased with increasing Staph. aureus concentrations. As expected, the recovery rate of DNA in milk-powder samples was slightly higher than was that in pork samples.

Generally, the enrichment of DNA by Si-MNP occurred via a nonspecific absorption process, so the presence of nontarget DNA in the sample partially affected the efficiency of the enrichment procedure. Therefore, the DNA present in pork tissue might competitively occupy the binding sites of Si-MNP, thereby lowering the lower capability for DNA enrichment. Therefore, we verified that the recovery rate of DNA in milkpowder samples was slightly higher than was that from pork samples in the current study. Compared with the traditional DNA extraction methods such as phenolchloroform extraction, the Si-MNP method avoids the time-consuming and laborious process of preculturing of the target pathogen. It also eliminates interference from components including ions, proteins, and fatty matter in samples.

\section{Specificity of the Protocol}

To assess the specificity of the protocol, 23 Staph. aureus and 27 non-Staph. aureus strains commonly used in food fermentation and frequent bacterial contaminants in food were amplified using tHDA and observed under UV light. As shown in Table 1, all 23 Staph. aureus 


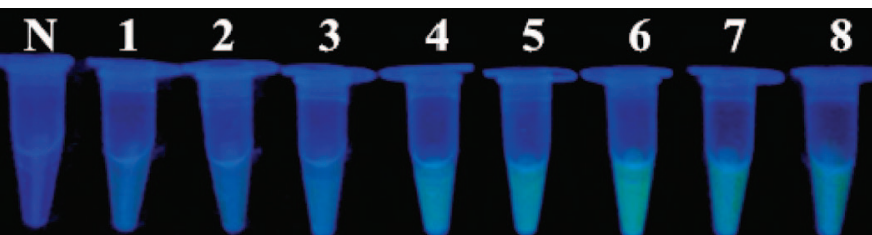

Figure 3. Detection limit of the Si-MNP-tHDA-SYBR Green I assay using serially diluted Staphylococcus aureus in pure culture. Lanes 1 to $8=$ from $5 \times 10^{0}$ to $5 \times 10^{7} \mathrm{cfu} / \mathrm{mL} ; \mathrm{N}=$ negative control. All samples were repeated in triplicate. Si-MNP $=$ silica-coated magnetic nanoparticles; tHDA $=$ thermophilic helicase-dependent isothermal amplification. SYBR Green I (Invitrogen, Shanghai, China). Color version available online.

strains gave positive signals, whereas the 27 non-Staph. aureus strains generated no signal. Therefore, the protocol appeared to be highly specific for the detection of Staph. aureus.

\section{Detecting Staph. aureus in Pure Cultures}

The performance of the novel protocol in pure cultures was evaluated using different concentrations of Staph. aureus (ranging from $10^{0}$ to $10^{7} \mathrm{cfu} / \mathrm{mL}$ ). As shown in Figure $3,10^{0} \mathrm{cfu} / \mathrm{mL}$ of Staph. aureus could be visualized under a UV lamp. The tHDA amplification products were also analyzed using gel electrophoresis; however, the maximum detection limit was only $10^{4}$ $\mathrm{cfu} / \mathrm{mL}$ (Figure 4).

The results of gel electrophoresis demonstrated that the target DNA was amplified successfully and that the current SYBR Green I-based DNA detection method

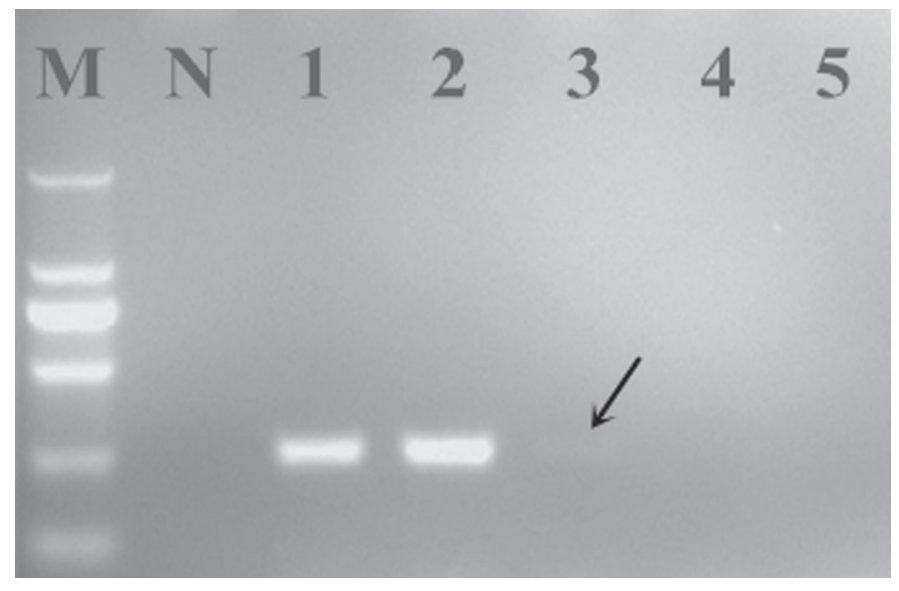

Figure 4. Detection limit of Si-MNP-tHDA-agarose gel electrophoresis using serially diluted Staphylococcus aureus in pure culture. Lane $\mathrm{M}=\mathrm{DL} 2000$ marker; lanes 1 to $5=$ from $5 \times 10^{5}$ to $5 \times 10^{1} \mathrm{cfu} /$ $\mathrm{mL} ; \mathrm{N}=$ negative control. Size of the target gene was about $263 \mathrm{bp}$. Si-MNP = silica-coated magnetic nanoparticles; tHDA $=$ thermophilic helicase-dependent isothermal amplification. The arrow indicates the detection limit by gel electrophoresis; the band is lane 3 is weak but real. was more sensitive than that of gel electrophoresis. Loop-mediated isothermal amplification, a most common isothermal amplification technique, was also used to detect Staph. aureus. It had a detection limit of 8 to $10 \mathrm{cfu} / \mathrm{mL}$ (Niessen et al., 2013), which was similar to that of the tHDA protocol. However, loop-mediated isothermal amplification assays normally require 6 primers, which increase the complexity of the experiment. Moreover, the novel assay did not require any special processing or electrophoresis to identify the positive reaction.

\section{Application of the Method in Spiked Milk Powder and Pork Samples}

To evaluate the feasibility of the assay for detecting Staph. aureus in food samples, milk powder and pork samples were spiked because of the lack of pathogens in these samples. As shown in Figure 5A, the detection limit in milk-powder samples spiked with mixture of $10^{8} \mathrm{cfu} / \mathrm{mL}$ of each E. coli, L. monocytogenes, and L. plantarum remained $10^{0} \mathrm{cfu} / \mathrm{mL}$ of Staph. aureus. Similarly, the detection limit was $10^{1} \mathrm{cfu} / \mathrm{mL}$ in pork samples (Figure 5B). These results indicate that a good specificity and sensitivity for the detection of Staph. aureus in food samples was achieved, even in the presence of interference from high concentrations of non-Staph. aureus bacteria.

The Si-MNP-tHDA-SYBR Green I assay used to detect Staph. aureus was more sensitive in milk powder compared with pork samples. This is consistent with

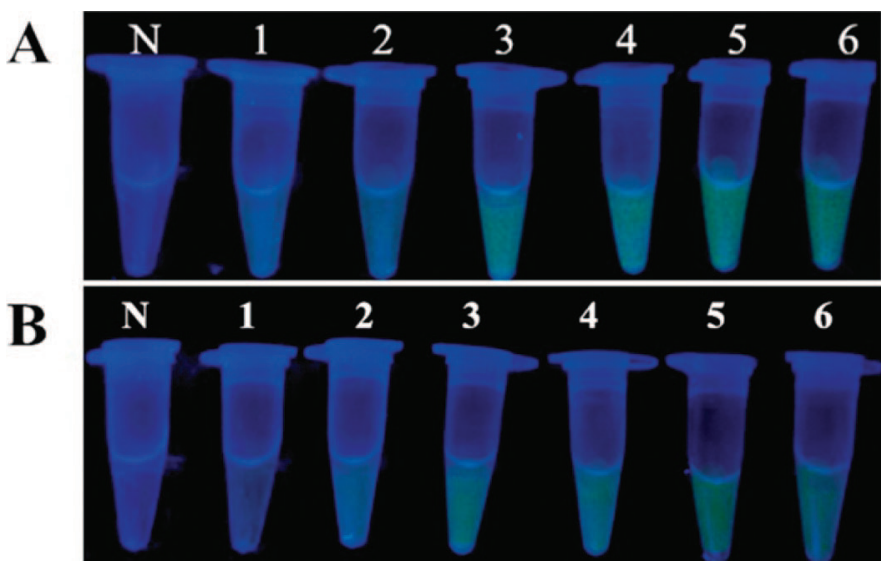

Figure 5. Detection limit of the Si-MNP-tHDA-SYBR Green I assay using serially diluted Staphylococcus aureus mixed with $10^{8} \mathrm{cfu} / \mathrm{mL}$ of nontarget bacteria cells (Escherichia coli, Listeria monocytogenes, and Lactobacillus plantarum) in powder milk samples (A) and pork samples (B). Lanes 1 to $6=$ from $5 \times 10^{0}$ to $5 \times 10^{5} \mathrm{cfu} / \mathrm{mL} ; \mathrm{N}=$ negative control. Si-MNP = silica-coated magnetic nanoparticles; tHDA $=$ thermophilic helicase-dependent isothermal amplification. SYBR Green I (Invitrogen, Shanghai, China). Color version available online. 

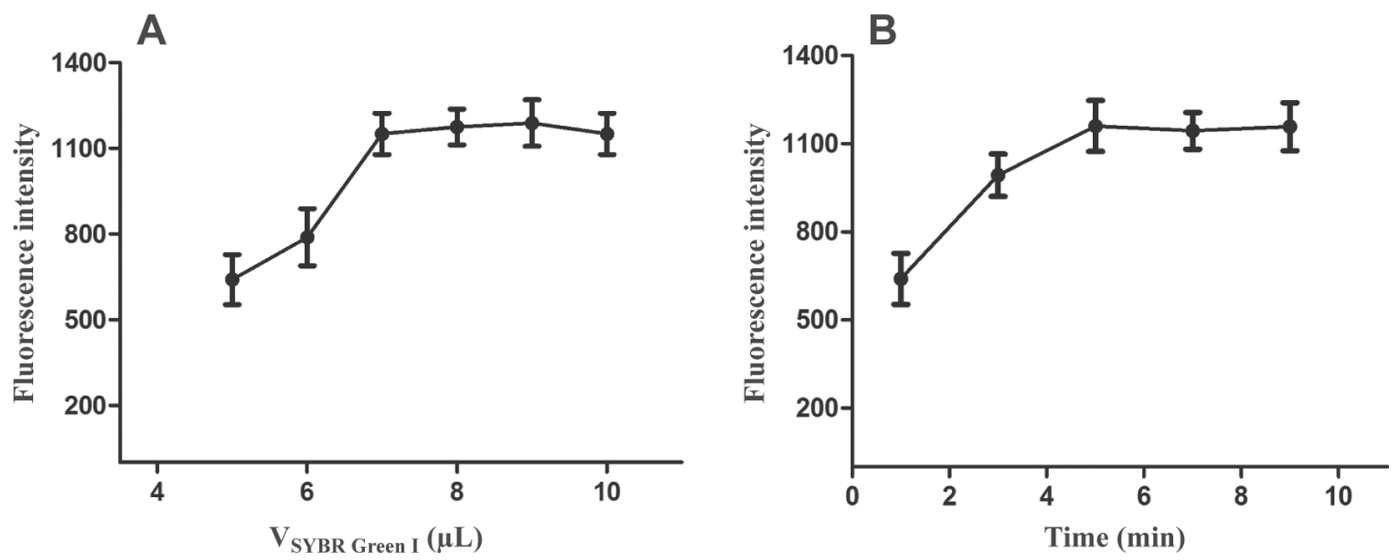

Figure 6. Influence of concentrations of SYBR Green I (Invitrogen, Shanghai, China; A) and incubation time (B) on the fluorescence intensity. Concentration of Staphylococcus aureus: $10^{8} \mathrm{cfu} / \mathrm{mL} . \mathrm{V}=$ the volume of SYBR Green I. Error bars indicate the deviation range in triplicate test results.

the recovery of DNA using Si-MNP in pork, which was lower than in milk-powder samples.

To investigate the universal applicability of this protocol further, 80 spiked milk powder and pork samples and 20 negative milk powder and pork samples were tested qualitatively. As shown in Table 2, sensitivities of 100 and $95 \%$ were obtained in milk powder and pork samples, respectively. Moreover, the results demonstrated that the protocol had a high specificity (100\%) for qualitative detection of Staph. aureus.

\section{Optimization of the Detection Conditions}

Because the tHDA protocol used the change in fluorescence of SYBR Green I bound to the dsDNA, the concentration of SYBR Green I might have affected the assay performance. To test this possibility, the effect of SYBR Green I concentration on the assay performance was evaluated. As shown in Figure 6A, the fluorescence intensity of SYBR Green I-dsDNA increased when the concentration of SYBR Green I was increased in the range from 5 to $7 \mu \mathrm{L}$; it then plateaued from 7 to 10 $\mu \mathrm{L}$. Therefore, $7 \mu \mathrm{L}$ of SYBR Green I was selected for subsequent assays. Similarly, the incubation time with SYBR Green I to detect Staph. aureus was optimized. As shown in Figure 6B, the fluorescence intensity increased greatly from 1 to 5 min and leveled off thereafter. Therefore, the optimized conditions $(7 \mu \mathrm{L}$ of SYBR Green I and $5 \mathrm{~min}$ ) were selected to achieve a satisfactory signal.

To determine the relationship between amplification time and fluorescence intensity in pure cultures, the tHDA reaction was performed using different amplification times. As shown in Figure 7, relatively good linearity was observed after 60 min of amplification $\left(\mathrm{R}^{2}=\right.$ 0.9970). Therefore, 60 min was selected for subsequent quantitative assays.

\section{Quantitative Detection Using the Novel Protocol}

The relationship between the SYBR Green I fluorescence intensity and the concentration of Staph. aureus was investigated further using the optimized conditions. As shown in Figure 8, the data showed a good linearity range between $10^{2}$ and $10^{4} \mathrm{cfu} / \mathrm{mL}$ of Staph. aureus. The fitted regression equation was $F=0.02225 C+$ 49.29 , where $F$ is the fluorescence intensity and $C$ is the concentration of Staph. aureus, with a reliable correlation coefficient $\left(\mathrm{R}^{2}=0.9972\right)$. Interestingly, the data could be fitted to a polynomial curve across a wide dynamic range (data not shown).

Furthermore, recovery studies were performed by spiking samples of milk powder and pork with different concentrations of Staph. aureus (ranging from $10^{2}$ to $10^{4}$

Table 2. Evaluation of Si-MNP-tHDA-SYBR Green I assay in spiked milk powder and pork samples ${ }^{1}$

\begin{tabular}{lccccc}
\hline & \multicolumn{2}{c}{ Sample size } & & \multicolumn{2}{c}{ Test results } \\
\cline { 2 - 3 } \cline { 5 - 6 } Sample & Positive & Negative & & Sensitivity (\%) & Specificity (\%) \\
\hline Milk powder & 80 & 20 & & 100 & 100 \\
Pork & 80 & 20 & & 95 & 100 \\
\hline
\end{tabular}

${ }^{1} \mathrm{Si}-\mathrm{MNP}=$ silica-coated magnetic nanoparticles; tHDA = thermophilic helicase-dependent isothermal amplification. SYBR Green I (Invitrogen, Shanghai, China). 


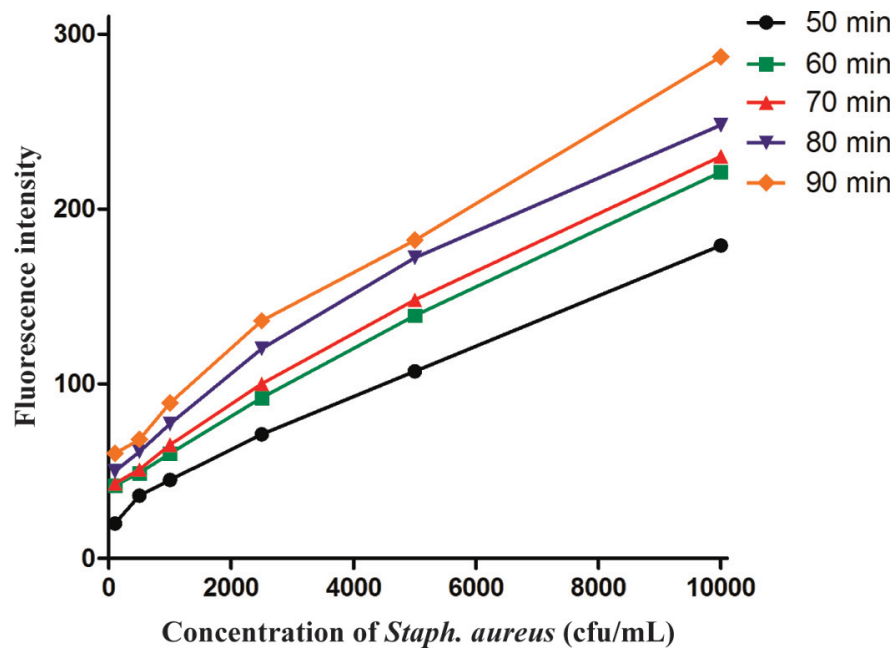

Figure 7. Influence of amplification time on the fluorescence intensity (cell counts of Staphylococcus aureus ranging from $1 \times 10^{2}$ to $1 \times$ $\left.10^{4} \mathrm{cfu} / \mathrm{mL}\right)$. Color version available online.

$\mathrm{cfu} / \mathrm{mL})$ to evaluate the reliability of the quantitative detection system. As shown in Figure 9, the Staph. aureus concentrations measured were very close to those added $(P>0.05)$. The results suggested that Staph. aureus concentrations from $10^{2}$ to $10^{4} \mathrm{cfu} / \mathrm{mL}$ could be determined quantitatively using the novel Si-MNPtHDA-SYBR Green I detection system.

The combination of helicase-dependent isothermal amplification and lateral flow provided an ideal platform for an assay to detect Staph. aureus with a detection limit of $2 \times 10^{4} \mathrm{cfu} / \mathrm{mL}$ in blood culture medium (Goldmeyer et al., 2008); however, this assay

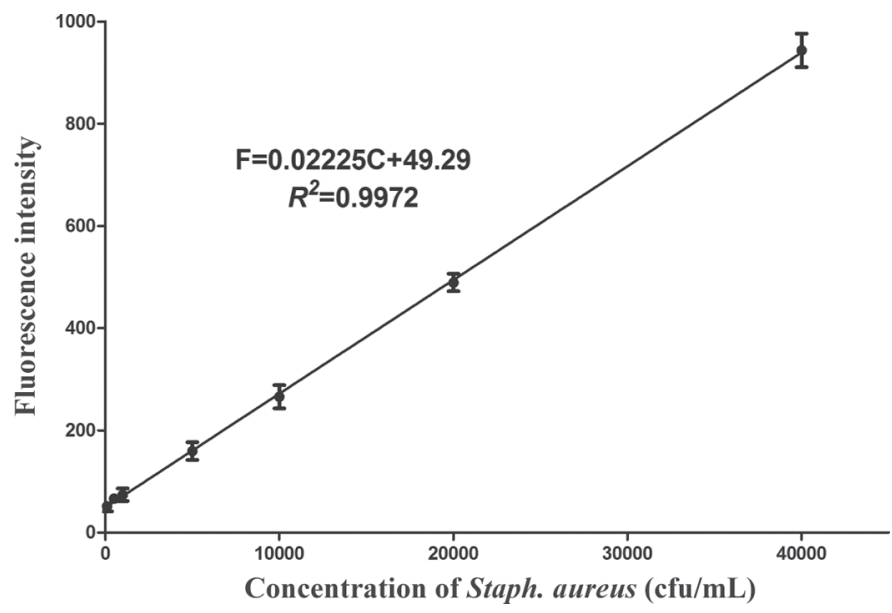

Figure 8. The linear relationship between the fluorescence intensity and the concentrations of Staphylococcus aureus (cell counts of Staph. aureus ranging from $1 \times 10^{2}$ to $4 \times 10^{4} \mathrm{cfu} / \mathrm{mL}$ ). Error bars indicate the deviation range in triplicate test results. $\mathrm{F}=$ fluorescence intensity, C = concentration of Staph. aureus.

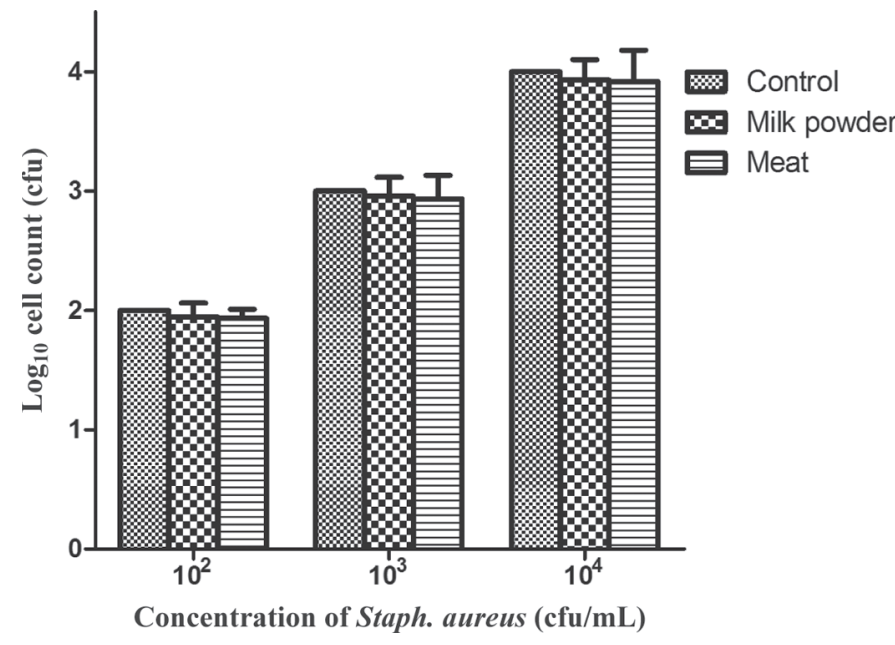

Figure 9. The recovery of Staphylococcus aureus in milk powder and pork samples detected by the Si-MNP-tHDA-SYBR Green I assay. $\mathrm{Si}-\mathrm{MNP}=$ silica-coated magnetic nanoparticles; $\mathrm{tHDA}=$ thermophilic helicase-dependent isothermal amplification. SYBR Green I (Invitrogen, Shanghai, China). Error bars indicated the deviation range in triplicate test results $(P>0.05)$.

could not be used for quantitative analysis. Compared with conventional PCR-based techniques, the novel SiMNP-tHDA-SYBR Green I method required a shorter sample preparation time, and the detection sensitivity was superior.

\section{CONCLUSIONS}

The present study describes the development and optimization of a Si-MNP-based tHDA assay to detect the specific DNA fragments of Staph. aureus using SYBR Green I. In the assay, expensive instrumentation was not required, and the results could be detected qualitatively by visual examination under UV lamp or quantitatively using a spectrophotometer. The entire detection time of the protocol was less than $2 \mathrm{~h}$, and its sensitivity and specificity were confirmed using spiked samples. The method shows a great promise for the detection of Staph. aureus in food samples. Furthermore, our Si-MNP-based tHDA detection strategy could provide an easy, convenient, and rapid method for sensing a wide range of pathogens with the use of appropriate primers.

\section{ACKNOWLEDGMENTS}

This project was sponsored by "Twelfth Five-Year Plan" for the National Science and Technology Support Program (2011BAK10B06, 2011BAK10B01, 2011BAK10B02), Natural Science Foundation (31260363, 31000048), the Ganpo Talent Engineering 555 Project (18000035), the Academic and Technical 
Leaders Training Program for Major Subjects of Jiangxi Province (2009-12), and the Research Program of the State Key Laboratory of Food Science and Technology of Nanchang University (SKLF-ZZA-201302, SKLF-TS-200916).

\section{REFERENCES}

Chen, F., X. Ming, X. Chen, M. Gan, B. Wang, F. Xu, and H. Wei. 2014a. Immunochromatographic strip for rapid detection of Cronobacter in powdered infant formula in combination with silica-coated magnetic nanoparticles separation and $16 \mathrm{~S}$ rRNA probe. Biosens. Bioelectron. 61:306-313.

Chen, X., M. Gan, H. Xu, F. Chen, X. Ming, H. Xu, H. Wei, F. Xu, and C. Liu. 2014b. Development of a rapid and sensitive quantum dot-based immunochromatographic strip by double labeling PCR products for detection of Staphylococcus aureus in food. Food Contr. 46:225-232.

Doseeva, V., T. Forbes, J. Wolff, Y. Khripin, D. O'Neil, T. Rothmann, and I. Nazarenko. 2011. Multiplex isothermal helicase-dependent amplification assay for detection of Chlamydia trachomatis and Neisseria gonorrhoeae. Diagn. Microbiol. Infect. Dis. 71:354-365.

Gilbert, G. L. 2002. Molecular diagnostics in infectious diseases and public health microbiology: Cottage industry to postgenomics. Trends Mol. Med. 8:280-287.

Gill, P., A.-H. Alvandi, H. Abdul-Tehrani, and M. Sadeghizadeh. 2008. Colorimetric detection of Helicobacter pylori DNA using isothermal helicase-dependent amplification and gold nanoparticle probes. Diagn. Microbiol. Infect. Dis. 62:119-124.

Gill, P., and A. Ghaemi. 2008. Nucleic acid isothermal amplification technologies - A review. Nucleosides Nucleotides Nucleic Acids $27: 224-243$.

Goldmeyer, J., H. Li, M. McCormac, S. Cook, C. Stratton, B. Lemieux, H. Kong, W. Tang, and Y. W. Tang. 2008. Identification of Staphylococcus aureus and determination of methicillin resistance directly from positive blood cultures by isothermal amplification and a disposable detection device. J. Clin. Microbiol. 46:1534-1536.

Haible, D., S. Kober, and H. Jeske. 2006. Rolling circle amplification revolutionizes diagnosis and genomics of geminiviruses. J. Virol. Methods 135:9-16.

Hellyer, T. J., and J. G. Nadeau. 2004. Strand displacement amplification: A versatile tool for molecular diagnostics. Expert Rev. Mol. Diagn. 4:251-261.

Kim, C.-H., M. Khan, D. Morin, W. Hurley, D. Tripathy, M. Kehrli Jr., A. Oluoch, and I. Kakoma. 2001. Optimization of the PCR for detection of Staphylococcus aureus nuc gene in bovine milk. J. Dairy Sci. 84:74-83.

Kim, J., and C. J. Easley. 2011. Isothermal DNA amplification in bioanalysis: Strategies and applications. Bioanalysis 3:227-239.

Lazcka, O., F. Campo, and F. X. Munoz. 2007. Pathogen detection: A perspective of traditional methods and biosensors. Biosens. Bioelectron. 22:1205-1217.

Li, M., Y.-L. Wong, L.-L. Jiang, K.-L. Wong, Y.-T. Wong, C. B.S. Lau, and P.-C. Shaw. 2013. Application of novel loop-medi- ated isothermal amplification (LAMP) for rapid authentication of the herbal tea ingredient Hedyotis diffusa Willd. Food Chem. 141:2522-2525.

Lowy, F. D. 1998. Staphylococcus aureus infections. N. Engl. J. Med. 339:520-532.

Mahalanabis, M., J. Do, H. A. L. Muayad, J. Y. Zhang, and C. M. Klapperich. 2010. An integrated disposable device for DNA extraction and helicase dependent amplification. Biomed. Microdevices 12:353-359.

Miao, T., Z. Wang, S. Li, and X. Wang. 2010. Sensitive fluorescent detection of Staphylococcus aureus using nanogold linked CdTe nanocrystals as signal amplification labels. Mikrochim. Acta 172:431-437.

Mollasalehi, H., and R. Yazdanparast. 2013. Development and evaluation of a novel nucleic acid sequence-based amplification method using one specific primer and one degenerate primer for simultaneous detection of Salmonella Enteritidis and Salmonella Typhimurium. Anal. Chim. Acta 770:169-174.

Niessen, L., J. Luo, C. Denschlag, and R. F. Vogel. 2013. The application of loop-mediated isothermal amplification (LAMP) in food testing for bacterial pathogens and fungal contaminants. Food Microbiol. 36:191-206.

Omiccioli, E., G. Amagliani, G. Brandi, and M. Magnani. 2009. A new platform for real-time PCR detection of Salmonella spp., Listeria monocytogenes and Escherichia coli $\mathrm{O} 157$ in milk. Food Microbiol. 26:615-622.

Picard, C., J. Fioramonti, A. Francois, T. Robinson, F. Neant, and C. Matuchansky. 2005. Review article: Bifidobacteria as probiotic agents-Physiological effects and clinical benefits. Aliment. Pharmacol. Ther. 22:495-512.

Scallan, E., R. M. Hoekstra, F. J. Angulo, R. V. Tauxe, M.-A. Widdowson, S. L. Roy, J. L. Jones, and P. M. Griffin. 2011. Foodborne illness acquired in the United States-Major pathogens. Emerg. Infect. Dis. 17:7-15.

Vancraeynest, D., F. Haesebrouck, and K. Hermans. 2007. Multiplex PCR assay for the detection of high virulence rabbit Staphylococcus aureus strains. Vet. Microbiol. 121:368-372.

Vincent, M., Y. Xu, and H. Kong. 2004. Helicase-dependent isothermal DNA amplification. EMBO Rep. 5:795-800.

Wang, C.-H., K.-Y. Lien, J.-J. Wu, and G.-B. Lee. 2011. A magnetic bead-based assay for the rapid detection of methicillin-resistant Staphylococcus aureus by using a microfluidic system with integrated loop-mediated isothermal amplification. Lab Chip 11:1521-1531.

Yang, A. K., H. Lu, S. Y. Wu, H. C. Kwok, H. P. Ho, S. Yu, A. K. Cheung, and S. K. Kong. 2013. Detection of Panton-Valentine Leukocidin DNA from methicillin-resistant Staphylococcus aureus by resistive pulse sensing and loop-mediated isothermal amplification with gold nanoparticles. Anal. Chim. Acta 782:46-53.

Yang, Y., X.-d. Su, Y.-w. Yuan, C.-y. Kang, Y.-j. Li, W. Zhang, and X.-y. Zhong. 2007. Detection of Staphylococcus aureus in dairy products by polymerase chain reaction assay. Agric. Sci. China 6:857-862. 